\title{
CICATRIZAÇÃO DA MIOTOMIA DE HELLER POR ACESSO VIDEOLAPAROSCÓPICO COM E SEM FUNDOCARDIOPLASTIA DE DOR ASSOCIADA, EM PORCOS
}

\author{
WOUND HEALING OF LAPAROSCOPIC HELLER'S MYOTOMY WITH AND WITHOUT \\ AN ADDED DOR'S FUNDOPLICATION, IN PIGS
}

\author{
João Luiz Moreira Coutinho Azevedo, TCBC-SP ${ }^{1}$; Fábio Okutani Kozu ${ }^{2}$; Otávio Azevedo ${ }^{3}$ \\ Vanessa Carla Paiva ${ }^{4}$; Cássio Edvan Paulino da Silva ${ }^{5}$; Manoel de Jesus Simões ${ }^{6}$
}

\begin{abstract}
RESUMO: Objetivo: Comparar a cicatrização da miotomia esofagiana laparoscópica associada ou não à fundocardioplastia de Dor. Métodos: Foram utilizados 18 porcos, em três grupos de seis animais. No grupo A foi realizada miotomia esofagiana. No grupo B associou-se plicatura gástrica à miotomia. O grupo C foi sem miotomia. No $21^{\circ}$ dia pós-operatório houve moldagem do lume para obter índices de estenose (IE) na região da miotomia (RM) e na transição esôfago-gástrica (I'E'). Foram também estudados aspectos macro e microscópicos. Resultados: Duração maior ( $t$ de Student) no grupo B (93,6 minutos) que no A (45 minutos). Considerando-se o IE dos grupos A e B, não houve estenose e eles se equivaleram - Mann-Withney (-11.1\% no grupo A e -12.7\% no grupo B). O I'E' foi sempre maior que o IE - Wilcoxon (18\% versus $-11,1 \%$ no grupo A e $37,7 \%$ versus $-12.7 \%$ no grupo B). O I'E' do grupo B (37,7\%) foi o maior entre os grupos (Kruskal-Wallis): A: 18\%; C: 15,5\%. Houve regularidade macroscópica da região da miotomia do grupo A e deformidades com inflamação aguda persistente e granulomas no B. No grupo A houve epitelização mesotelial e no B a superfície cruenta permaneceu granulada. Na RM do grupo B, leucócitos (22 versus 8,6 do A) e vasos (18,7 versus 9,7 da A) foram mais numerosos. A fibrose foi mais profusa no grupo B (25,85 fibras versus 15,6 no A). Conclusões: A plicatura gástrica sobre a miotomia esofagiana propicia cicatrização menos adequada e é mais demorada que a miotomia isolada (Rev. Col. Bras. Cir. 2004; 31(6): 349-354).
\end{abstract}

Descritores: Acalasia Esofágica; Laparoscopia; Cicatrização; Cirurgia Vídeo-Assistida; Procedimentos Cirúrgicos Operatórios; Suínos.

\section{INTRODUÇÃO}

A extensão da esôfago-cárdio-miotomia de HellerZaaijer (Heller, 1913' Z Zaaijer, 1923²) no tratamento da acalásia esofagiana deve ser suficiente para secionar todas as fibras da camada muscular dos oito centímetros distais do esôfago e dos três centímetros proximais do estômago, de modo a ser eliminado por inteiro o obstáculo intrínseco da junção esôfago-gástrica à progressão do bolo alimentar ${ }^{3,4}$. Tal incisão suprime por completo uma importante barreira anti-refluxo: o mecanismo esfinctérico intrínseco do esôfago inferior. Em decorrência, prevenir o refluxo gastroesofagiano iatrogênico após miotomia é preocupação dos cirurgiões desde Heller: "O esfíncter após miotomia deve ser suficientemente débil para permitir a passagem dos alimentos, mas não a ponto de permitir o refluxo ${ }^{1}$.

Na cirurgia convencional, é quase unânime a recomendação de se adicionar um procedimento anti-refluxo à miotomia ${ }^{4}$. Com o advento da cirurgia minimamente invasiva, a miotomia esofagiana passou do campo das intervenções convencionais para o âmbito da videocirurgia ${ }^{5-7}$, preferencialmente videolaparoscopia ${ }^{8}$, dessa forma elidindo a dilatação pneumática como tratamento primário da acalásia nos estágios iniciais ${ }^{9}$.

Entretanto, mesmo por videocirurgia, muitos recomendam a associação de procedimento anti-refluxo após miotomia $^{\mathbf{1 0}}$, sendo a fundocardioplastia parcial anterior de Dor, descrita originariamente para cirurgia aberta ${ }^{11}$ e adaptada para o acesso laparoscópico ${ }^{12}$, a mais utilizada ${ }^{13-19}$.

A recomendação da associação de plicatura gástrica sobre a zona cruenta da miotomia esofagiana reporta-se à cirurgia convencional ${ }^{20,21}$. Nesse universo argumenta-se que, além de prevenir o refluxo gastresofagiano iatrogênico, o fundo gástrico plicado sobre a zona cruenta - tamponando perfurações inadvertidas do esôfago e interpondo-se entre as bordas da ferida - evitaria fatores determinantes da recidiva da acalásia, tais como estenose por fibrose periesofagiana que se seguem a escapes despercebidos de conteúdo

1. Professor Adjunto Mestre e Doutor do Departamento de Cirurgia e Responsável pelo Setor de Videocirurgia da TOCE-UNIFESP; Orientador (Mestrado e Doutorado) do Programa de Pós-Graduação em Cirurgia e Experimentação; Lider do Grupo "Videocirurgia" do CNPq/UNIFESP; Membro Titular da Sociedade Brasileira de Cirurgia Laparoscópica.

2. Mestre do Programa de Pós-Graduação em Cirurgia e Experimentação da UNIFESP- Pesquisador do CNPq

3. Médico-Assistente de Gastrocirurgia do Hospital do Servidor Público do Estado de São Paulo; Mestrando do Programa de Pós-Graduação em Cirurgia e Experimentação da UNIFESP - Pesquisador do CNPq

4. Mestranda do Programa de Pós-Graduação em Cirurgia e Experimentação da UNIFESP-EPM - Pesquisador do CNPq

5. Monitor da Disciplina de Técnica Operatória e Cirurgia Experimental da UNIFESP-EPM - Pesquisador do CNPq

6. Professor Livre-Docente do Departamento de Morfologia e Chefe de Disciplina de Histologia da UNIFESP

Recebido em 19/04/2004

Aceito para publicação em 03/09/2004

Trabalho realizado no Laboratório do Setor de Videocirurgia da Disciplina de Técnica Operatória e Cirurgia Experimental (TOCE), Departamento de Cirurgia da Universidade Federal de São Paulo (UNIFESP), Programa de Pós-Graduação em Cirurgia e Experimentação. 
esofagiano e reconstituição do mecanismo esfinctérico intrínseco do esôfago inferior por coaptação entre si das bordas da ferida ${ }^{22,23}$. Entretanto, mesmo fora do âmbito da cirurgia convencional, cirurgiões laparoscopistas que associam a fundocardioplastia de Dor à miotomia adotam tais argumen$\operatorname{tos}^{24}$.

Noutra ordem de idéias, há autores que consideram peculiaridades tais no acesso laparoscópico para o tratamento da acalásia que julgam poder prescindir da associação de qualquer procedimento anti-refluxo à miotomia laparoscópica, em casos selecionados ${ }^{25}$. Há publicações de séries de casos submetidos à operação de Heller laparoscópica sem procedimento anti-refluxo, com bons resultados ${ }^{26-31}$

Alegações em contrário ${ }^{32}$ aos argüidos benefícios da plicatura da parede gástrica sobre a superfície cruenta da miotomia dizem respeito à dificuldade da reabsorção do exsudato inflamatório circunscrito pelo fundo gástrico plicado, cuja permanência levaria a aumento da fibrose na região. Em peritônio livre, essas coleções seriam prontamente absorvidas, propiciando epitelização da zona cruenta por células mesoteliais, caso essa não estivesse isolada do líquido peritoneal por intermédio do fundo gástrico plicado sobre ela. Adicionalmente, a magnificação do campo operatório videocirúrgico evidenciaria microperfurações iatrogênicas que identificadas mediante teste de injeção de azul de metileno e gás - não têm como passar despercebidas. Tenha-se ainda em mente que o tônus muscular da camada circular da musculatura esofágica seccionada tende a condicionar o afastamento uma da outra das bordas da miotomia, de forma a não haver risco de sua coaptação $0^{3,25}$.

A hipótese aqui testada é a de que a superfície cruenta resultante da esôfago-cárdio-miotomia, permanecendo exposta na técnica sem válvula associada, é epitelizada por células mesoteliais livres no líquido peritoneal, resultando em excelente reparo da miotomia, com manutenção adequada do afastamento das bordas musculares secionadas no procedimento.

Dessa forma, o objetivo desta pesquisa é comparar a cicatrização da miotomia esofagiana (operação de HellerZaaijer) por via laparoscópica com e sem fundocardioplastia de Dor associada.

\section{MÉTODO}

Foram utilizados 18 porcos machos da raça Large White, com peso corpóreo entre 15 a $20 \mathrm{~kg}$, distribuídos aleatoriamente em grupo A (miotomia isolada), grupo B (miotomia associada a fundocardioplastia anterior) e grupo C (sem intervenção sobre o esôfago). Antes da operação os animais ficavam em jejum alimentar por 18 horas e jejum hídrico por 8 horas.

Os animais foram anestesiados, com intubação orotraqueal e colocados sob pressão positiva intermitente. Para tanto receberam por via intramuscular uma associação de quetamina na dose de 30mg/kg com acepromazina na dose de $0,5 \mathrm{mg} / \mathrm{kg}$ como medicação pré-anestésica. Para indução anestésica os animais receberam por via intravenosa tiopental na dose de $12 \mathrm{mg} / \mathrm{kg}$ juntamente com brometo de pancurônio na dose de 0,066mg/kg. A manutenção do plano anestésico foi feita com vaporização de isofluorano a $2 \%$ e citrato de fentanila na dose de $0,005 \mathrm{mg} / \mathrm{kg}$ administradas por via intravenosa.

No pós-operatório imediato os animais receberam quetoprofeno por via intravenosa na dose de $2 \mathrm{mg} / \mathrm{kg}$.

O acesso operatório utilizado foi o videolaparoscópico em todos os animais, com cinco portais em posição usual para o acesso à junção esôfago-gástrica.

Nos grupos A e B, após a abertura do ligamento gastrofrênico e a demarcação a bisturi elétrico da localização e da extensão da miotomia, incisou-se a face anterior do esôfago numa extensão de $5 \mathrm{~cm}$ acima do esfíncter inferior, interessando as túnicas musculares. Nos animais do grupo B foi interposto um segmento da parede anterior do fundo gástrico, plicado sobre a superfície cruenta da miotomia e fixado às bordas cruentas da musculatura esofagiana seccionada mediante três pontos simples de Poliéster 3-0. Nos animais do grupo C foi estabelecido o pneumoperitônio, colocados os trocartes e nenhuma intervenção sobre o esôfago ocorreu.

Ao final do procedimento cirúrgico estabeleceu-se um período de 24 horas de jejum pós-operatório no que diz respeito ao fornecimento de ração. A vasilha de água era colocada à disposição do animal logo após a recuperação anestésica. A partir do primeiro dia pós-operatório os animais receberam ração industrializada fornecida quatro vezes por dia e água ad libitum.

Os animais foram observados no período pós-operatório, com avaliação do estado geral e do sistema digestório e com vistas especiais para eventuais sinais de distúrbios do trânsito alimentar no esôfago.

No $21^{\circ}$ dia pós-operatório os animais foram mortos através da utilização de quetamina na dose de $30 \mathrm{mg} / \mathrm{kg}$ com acepromazina na dose de $0,5 \mathrm{mg} / \mathrm{kg}$ por via intramuscular. Uma vez manifestados os efeitos dessa associação, ministrou-se um combinado de embutamina, iodeto benzônico e cloridrato de tetracaína por via intravenosa na dose de $0,3 \mathrm{ml} /$ $\mathrm{kg}$. Em seguida foi retirado um segmento esofagiano de $20 \mathrm{~cm}$, compreendendo a transição esôfago-gástrica (TEG) e abrangendo a região da miotomia (RM), do qual foram retiradas amostras para exame histopatológico.

Três padrões morfológicos microscópicos foram definidos para fins de avaliação morfométrica: leucócitos (constituído por células polimorfonucleadas e células mononucleadas), vasos neoformados e substância intercelular (fibras colágenas).

Para a moldagem do lume das vísceras, injetou-se 60 ml de uma solução de hidrocolóide irreversível, composta de diatomita, alginato de potássio, sulfato de cálcio, carbonato de magnésio, aromas e corantes. Após o seu endurecimento, o molde foi retirado do lume esofágico para a determinação dos índices de estenose da RM (IE) e da TEG (I'E').

Para avaliar o grau de estenose do lume esofagiano, foi utilizado o molde da peça visando à aferição do perímetro interno do lume do esôfago.

Para a determinação do IE foram mensurados os perímetros externos do molde na RM (A) e na região correspondente a $2 \mathrm{~cm}$ craniais $(\mathrm{B})$ e a $2 \mathrm{~cm}$ caudais $(\mathrm{C})$ às extremidades longitudinais da miotomia. Destas três medidas foi obtido o 
IE, segundo a fórmula (McAdams et $a^{33}$ ) IE = $100(1-2 \mathrm{~A} /$ $\mathrm{B}+\mathrm{C})$.

Para a determinação do I'E' foram medidos os perímetros externos do molde na TEG (A') e em outros dois pontos situados cranialmente à mesma, sendo um deles (C') a $2 \mathrm{~cm}$ e o outro (B') a 4cm. Para a determinação do I'E', utilizou-se a mesma fórmula de McAdams ${ }^{33}$.

Foi investigada a presença ou não de aderências peritoneais na região da miotomia.

Para análise dos resultados foi aplicado o teste $t$ de Student, o teste de Wilcoxon, o teste de Mann-Whitney e a análise de variância de Kruskal-Wallis. Fixou-se em 0,05 ou $5 \%(\alpha \leq 0,05)$ o nível de rejeição da hipótese de nulidade assinalando-se com um asterisco os valores significantes.

\section{RESULTADOS}

Os animais de ambos os grupos não apresentaram sinais de dificuldade evidente na deglutição dos alimentos ou qualquer outro sinal clínico que pudesse levar a acreditar que os mesmos tivessem qualquer alteração do trânsito do bolo alimentar pelo esôfago.

A duração das intervenções operatórias no grupo com miotomia isolada (grupo A, 45 minutos) foi significantemente menor que no grupo B (93 minutos), que teve fundocardioplastia associada.

À necropsia, foi observado que os animais dos grupos A e B indistintamente apresentavam aderências peritoneais entre a região operada e a face visceral do fígado. O aspecto macroscópico na região da miotomia nos animais do grupo A era regular e evidenciava ausência de fibrose (Figura 1), enquanto que nos animais do grupo B notava-se fibrose profusa na transição esôfago-gástrica (Figura 2)

No estudo histológico qualitativo dos animais do grupo A, notou-se processo inflamatório discreto e ausência da túnica muscular do esôfago na região da miotomia, que foi substituída por tecido de reparação, recoberto por células de revestimento mesotelial, as quais puderam ser melhor visibilizadas em corte histológico tangencial à superfície cruenta da miotomia.

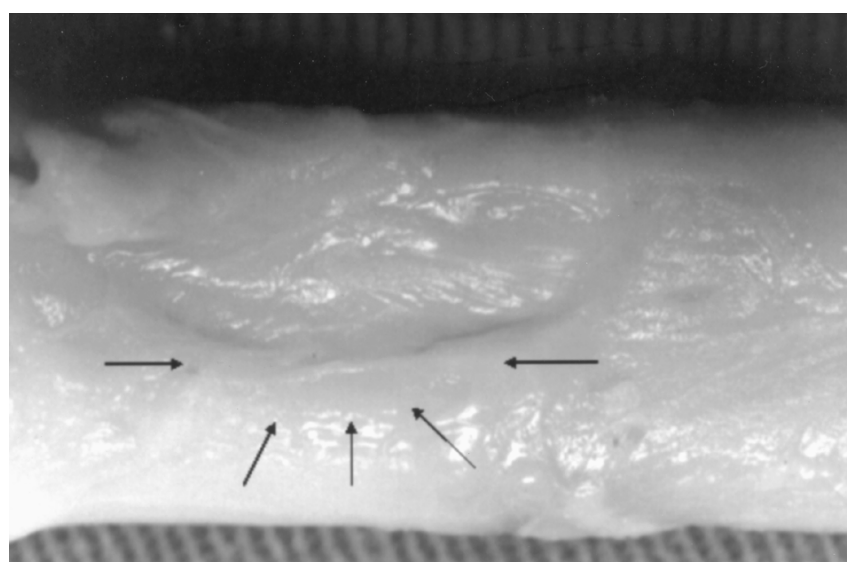

Figura 1- Fotografia da região miotomizada - setas (grupo A), constatando-se depressão acentuada no local da incisão cirúrgica do esôfago, com superfície regular e de aspecto nacarado.
Nos animais do grupo B foi encontrado intenso infiltrado linfoplasmocitário no local da miotomia, com tecido de granulação da superfície cruenta rico em capilares, leucócitos (linfócitos, neutrófilos e plasmócitos), macrófagos e fibras colágenas, não se notando epitelização por células mesoteliais.

A análise estatística (teste t de Student) demonstrou que os elementos da inflamação considerados no estudo morfométrico (leucócitos, vasos neoformados e fibras colágenas) foram significantemente mais numerosos (Gráfico 1) após miotomia com fundocardioplastia (grupo B) que com a miotomia isolada (grupo A).

Os índices de estenose na região da miotomia (IE) foram sempre negativos estatisticamente equivalentes entre si (teste de Wilcoxon) e menores que os indices de estenose (I'E') da transição esôfago- gástrica, indicando que houve aumento idêntico da amplitude do lumen do esôfago tanto na região onde foi realizada a miotomia isolada quanto na região da miotomia com adição da plicatura gástrica (Tabela 1; Gráfico 2).

A nível da transição esôfago-gástrica, ocorreu diminuição estatisticamente significante do lume esofágico (teste de Mann-Whitney) nos animais do grupo B quando comparados aos do grupo A (Tabela 1).

Adicionalmente, o índice de estenose da transição esôfago-gástrica dos animais submetidos a miotomia com associação de fundocardioplastia (grupo B) foi o maior dentre todos os grupos (Tabela 2; Gráfico 2 e Figura 3).

\section{DISCUSSÃO}

A propósito da pertinência ou não da associação de procedimento anti-refluxo à miotomia esofagiana laparoscópica no tratamento da acalásia, recente metanálise ${ }^{34}$ concluiu: “Baseados nos dados publicados, não podem ser feitas recomendações no sentido da adição de procedimento anti-refluxo à miotomia de Heller laparoscópica”.

Quanto à adequação que a fundocardioplastia de Dor associada à miotomia traria à cicatrização da região da miotomia, impedindo escapes e evitando coaptação das bordas da incisão muscular, a presente pesquisa pretendeu contribuir para o esclarecimento da forma mediante a qual o repa-

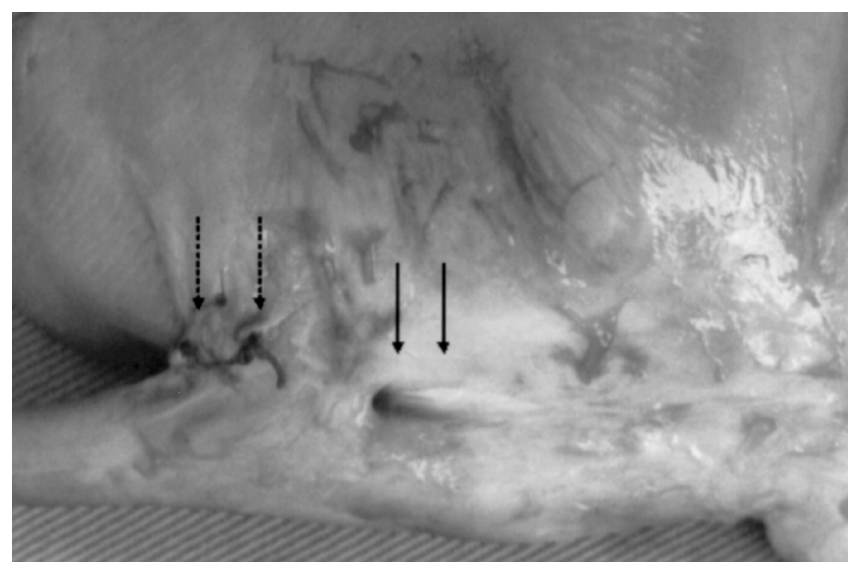

Figura 2 - Fotografia de plicatura gástrica sobre a região da miotomia (grupo B), evidenciando fios de sutura (setas pontilhadas) e profusa fibrose envolvendo a transição esôfago-gástrica (setas contínuas). 


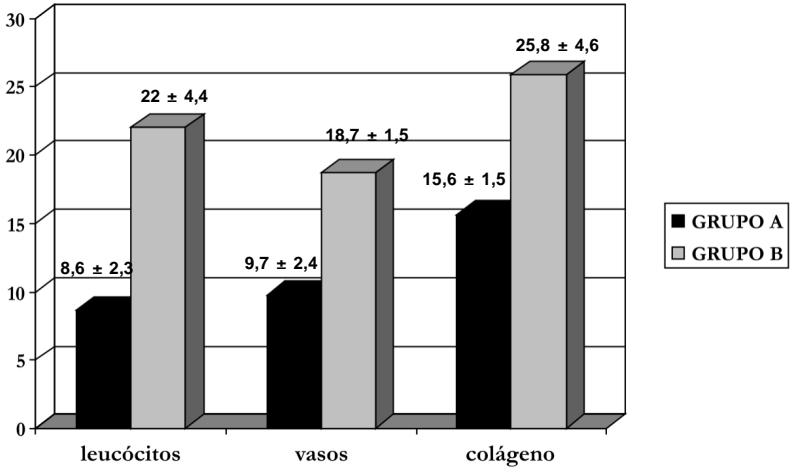

Teste t de Student

(Grupo A X Grupo B)

t crítico $=204$

$$
\begin{aligned}
& \text { Leucócitos } \\
& \text { t calc. }=13,35^{*} \\
& (\mathrm{P}<0,000) \\
& \mathrm{B}>\mathrm{A}
\end{aligned}
$$

$$
\begin{aligned}
& \text { F. Colágenas } \\
& \text { t calc. }=9,30^{*} \\
& (\mathrm{P}<0,000) \\
& \mathrm{B}>\mathrm{A}
\end{aligned}
$$

Gráfico 1 - Representação gráfica do número dos elementos da inflamação contados em 20 lâminas de cada grupo, com as respectivas médias e desvios-padrão, sendo os elementos contados no grupo com fundocardioplastia significantemente mais numerosos que os do com miotomia isolada.

Tabela 1- Índices de estenose na região da miotomia (IE) e da transição esôfago-gástrica (I'E') dos grupos A e B.

\begin{tabular}{lcccc}
\hline & \multicolumn{2}{c}{ GrupoA } & \multicolumn{2}{c}{ Grupo B } \\
\cline { 2 - 5 } & \multicolumn{1}{c}{ IE } & I'E' & IE & I'E' \\
\hline & $-4,76$ & 4,80 & $-6,74$ & 31,50 \\
& $-5,31$ & 24,50 & $-13,20$ & 43,40 \\
& $-11,76$ & 29,50 & $-12,22$ & 37,20 \\
& $-18,96$ & 12,50 & $-28,57$ & 28,60 \\
& $-14,28$ & 9,60 & $-8,10$ & 33,80 \\
Médias & $-11,60$ & 27,10 & $-4,76$ & 51,50 \\
Medianas & $-11,1$ & 18,0 & $-12,7$ & 37,7 \\
\hline
\end{tabular}

Teste de Wilcoxon

(Miotomia X Teg)

GrupoA

$$
\begin{gathered}
\text { T calc. }=0,0^{*}(\mathrm{P}<0,05) \\
\text { T crítico }=1,0
\end{gathered}
$$

I'E'>IE

Teste de Mann Whitney

(Grupo A X Grupo B)

IE

U calc. $=17,5$
U crít. $=5,0$
N.S.

I'E'

$$
\begin{gathered}
\text { U calc. }=1,0^{*}(\mathrm{P}<0,05) \\
\mathrm{U} \text { crít. }=5,0 \\
\mathrm{~B}>\mathrm{A}
\end{gathered}
$$

ro da superfície cruenta da miotomia esofagiana se processa na presença de duas circunstâncias distintas: deixada livre na cavidade peritoneal ou recoberta por fundo gástrico plicado sobre ela, como na técnica de Dor.

Pretendeu-se testar a hipótese de que a superfície cruenta da miotomia esofagiana - deixada cicatrizar sem a interferência do fundo gástrico plicado sobre ela - tende a apresentar pequeno processo inflamatório e receber a epitelização regular das células mesoteliais, oriundas da diferenciação e proliferação de células mesenquimais primitivas que existem livres no líquido peritoneal, conforme sói acontecer nas superfícies cruentas em geral banhadas por esse líquido.

Há autores que referem recidiva da acalásia por causa de cicatrização da área da miotomia ${ }^{22,35}$. Entretanto, depreende-se da análise da casuística desses autores que não houve uma nítida diferenciação histopatológica entre o refazimento do esfíncter inferior do esôfago e a estenose por estreitamento extrínseco do esôfago distal, mercê de fibrose.

A observação macroscópica e microscópica da região da miotomia dos animais operados nesta pesquisa mostrou que não houve reconstituição do esfíncter, em nenhum dos dois grupos. O que ocorreu foi um estreitamento extrínseco do esôfago operado, situação macroscopicamente indistinguível do simples refazimento do esfíncter esofágico inferior. Foi constatada, ainda, a perfeita cicatrização da miotomia sem válvula (Figura 1). Esses achados nos levam a conjeturar que, na casuística desses autores ${ }^{22,35}$, o que de fato ocorreu foi fibrose da transição esôfago-gástrica decorrente do fundo gástrico plicado. Tudo se passa como se a parede gástrica plicada sobre a superfície cruenta e suturada às bordas da miotomia funcionasse como poderoso indutor de fibrose (Figura 2 e Gráfico 1), com conseqüente estreitamento extrínseco da transição esôfago-gástrica (Figura 3).

Índice de estenose (percentual de redução do lume esofágico) igual a cem é equivalente à estenose total e IE igual a zero é equivalente a nenhuma estenose.

Nessa ordem de idéias, pudemos constatar que todos os índices de estenose da região da miotomia nesta pesquisa foram negativos (Tabela 1 e Gráfico 2), o que equivale a dizer que a miotomia propiciou um aumento do lume do órgão no local.

Tabela 2 - Índices de estenose ao nível da transição esôfagogástrica.

\begin{tabular}{cccc}
\hline & GrupoA & Grupo B & Grupo C \\
\hline & 4,8 & 31,5 & 10,5 \\
& 24,5 & 43,4 & 15,7 \\
& 29,5 & 37,2 & 9,7 \\
& 12,5 & 28,6 & 17,7 \\
& 9,6 & 33,8 & 19,8 \\
& 27,1 & 51,5 & 19,5 \\
\hline Médias & 18,0 & 37,6 & 15,5 \\
\hline
\end{tabular}

Análise de variância de Kruskal-Wallis

H calculado $=10,81^{*}(\mathrm{P}<0,01)$ H crítico $=5,99$

Grupo B > Grupo A e Grupo C 


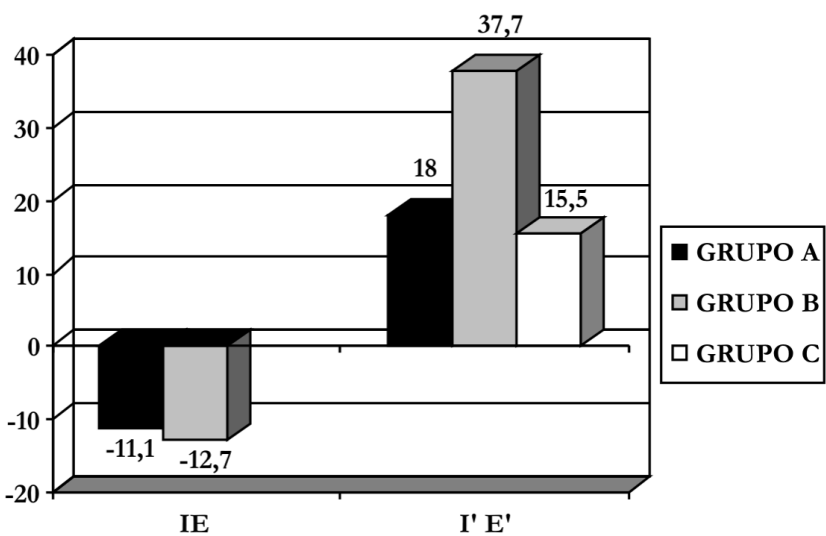

Gráfico 2 - Representação gráfica das médias dos índices de estenose na região da miotomia (IE) nos animais dos grupos $A$ e $B$ e na transição esôfago-gástrica (I'E') nos animais dos grupos $A, B$ e C.

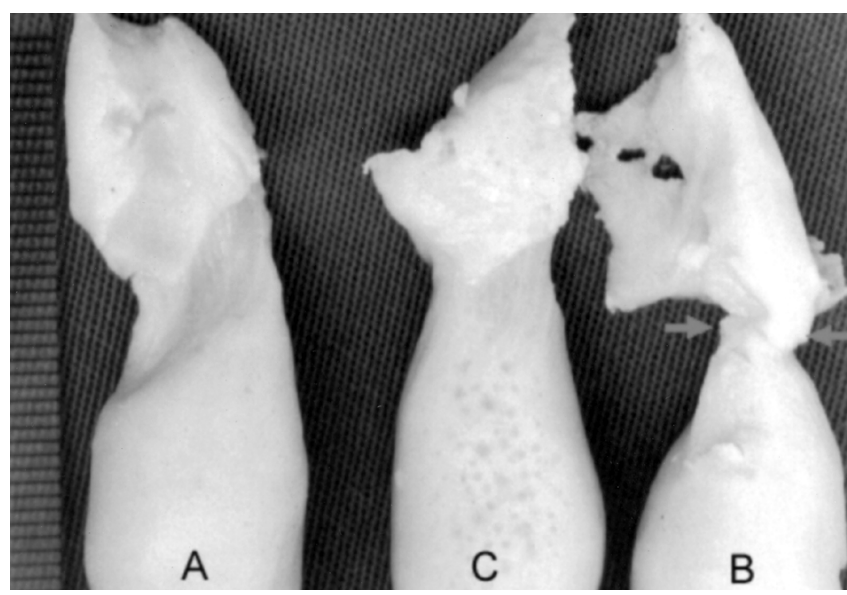

Figura 3 - Fotografia de moldes do lume esofagiano de animais dos grupos A, C e B (da esquerda para a direita), evidenciando diminuição acentuada do perímetro a nível da transição esôfago-gástrica do animal do grupo B (setas).

Dessa forma, este experimento não sustentou a teoria do refazimento da continuidade da musculatura esofagiana após miotomia sem plicatura gástrica aposta, contrariando assim a opinião de alguns autores ${ }^{22,35}$.
Por outro lado, a miotomia com plicatura gástrica (grupo B) também não evidenciou estenose no local - IE negativo (Tabela 1 e Gráfico 2).

Entretanto, na transição esôfago-gástrica, houve evidente diminuição da amplitude do lume esofagiano nos animais do grupo B, com plicatura, quando comparados com o grupo A, sem plicatura, e com os animais do grupo C, controles (Tabela 2, Gráfico 2 e Figura 3).

Tudo isso aponta para a conclusão de que os relatos dos autores ${ }^{22,35}$ que referem estenose esofagiana provavelmente dizem respeito a miotomias originariamente incompletas e não a uma coaptação entre si das bordas de uma miotomia completa.

Observou-se ainda nesta pesquisa que a adição do fundo gástrico plicado sobre a superfície cruenta, produz processo inflamatório de magnitude maior e também maior quantidade de fibrose (grupo B) - Figura 2 e Gráfico 1 - do que o processo cicatricial resultante da miotomia isolada (grupo A) Figura 1 e Gráfico1.

Finalmente, mas não menos importante, ficou claramente evidenciado que a epitelização da zona cruenta da miotomia isolada se fez de forma regular e constante em todos os animais do grupo A. Isso significa que o processo cicatricial resultou numa reconstituição adequada da superfície esofagiana (Figura 1).

Enfatize-se aqui que a presente pesquisa é a primeira experiência a ser feita com características tais que poderia ter surpreendido aspectos histopatológicos compatíveis com coaptação entre si das bordas musculares da miotomia. Entretanto, isso não ocorreu. Ao contrário, parece ter sido cabalmente demonstrado que a continuidade da musculatura esofagiana não se refaz após miotomia.

\section{AGRADECIMENTOS}

Agradecemos o suporte financeiro que nos concedeu o Fundo de Auxílio aos Docentes e Alunos (FADA) da Universidade Federal de São Paulo - Escola Paulista de Medicina e os equipamentos e materiais laparoscópicos que nos foram cedidos pela PCE - Pró-Cirurgia Especializada Ltda.

\begin{abstract}
Background: It has been argued that a Dor's fundoplication following myotomy is useful to prevent leakage due to overlooked iatrogenic perforations of esophagus and it is able to stop sphincter reconstruction, avoiding recurrent achalasia. Another strain of thought is that anterior fundoplication causes significant local distortion by fibrosis because the gastric patch impairs mesotelial epithelization by covering the myotomy and that iatrogenic perforations are easily diagnosed by laparoscopic magnification. The purpose of this research is to compare the wound healing of the laparoscopic esophageal myotomy with and without a gastric patch. Methods: Eighteen male pigs were distributed into three groups of six. Esophageal myotomy was performed in group A. A gastric patch was associated to group B. Myotomy was not performed in group C. On the 21st postoperative day, lumen molding was accomplished to determine the index of stenosis (IS) at the area of myotomy (AM) and at the oesophagogastric junction (OJ) Results: Longer operative duration (t Student) in group B (93. 6”) than in group A (45”). At AM, IS was negative (lumen increased) and equivalent in both groups (Mann-Withney): $-11.1 \%$ in group A and $-12.7 \%$ in group B. IS at OJ was always higher than IS at AM (Wilcoxon): $18 \%$ versus $-11.1 \%$ in group A and $37.7 \%$ versus $-12.7 \%$ in group B. IS at OJ in group B (37.7\%) was predominant among all groups (Kruskal-Wallis): group $A=18 \%$; group $C=15.5 \%$. Mesotelial epithelium was observed in group A. Inflammatory reaction was greater in group B (leucocytes: 22 versus 8.6; fibrosis: 25.5 fibers versus 15.6; and granulation tissue: 18.7 vessels versus 9.7) than in group A. Conclusion: Esophageal myotomy followed by Dor's fundoplication does not heal adequately and also results in lumen stricture at the oesophagogastric transition. Myotomy without gastric patch is faster and causes less inflammation.
\end{abstract}




\section{REFERÊNCIAS}

1. Heller E - Extramuköse Cardiaplastik beim chronischen Cardiospasmus mit Dilatation des Oesophagus. Mitt Grenzgeb Med Chir, 1913, 27:141-149.

2. Zaaijer JH - Cardiospasm in the aged. Ann Surg, 1923, 77:615617.

3. Azevedo JLMC, Boulez J - Technical systematization for laparoscopic esophageal myotomy (modified Heller's operation) without antireflux procedure. Surg Endosc, 2000, 14 (Suppl 1): S11 (Vídeo disponível em: URL: http:// www.cirurgiaonlinemed.br/heller.wmv).

4. Andreollo NA, Earlam RJ - Heller's myotomy for achalasia: is an added anti-reflux procedure necessary? Br J Surg, 1987, 74(9):765-769

5. Dempsey DT, Kalan MM, Gerson RS, et al. - Comparison of outcomes following open and laparoscopic esophagomyotomy for achalasia. Surg Endosc, 1999, 13(8):747-750

6. Shimi S, Nathanson LK, Cushieri A - Laparoscopic cardiomyotomy for achalasia. J R Coll Surg Edinb, 1991, 36(3):152-154

7. Pellegrini C, Wetter LA, Patti M, et al. - Thoracoscopic esophagomyotomy. Initial experience with a new approach for the treatment of achalasia. Ann Surg, 1992, 216(3):291-299.

8. Ramacciato G, Mercantini P, Amodio PM, et al. - The laparoscopic approach with antireflux surgery is superior to the thoracoscopic approach for the treatment of esophageal achalasia. Experience of a single surgical unit. Surg Endosc, 2002, 16(10):1431-1437.

9. Peillon C, Fromont G, Auvray S, et al. - Achalasia: the case for primary laparoscopic treatment. Surg Laparosc Endosc Percutan Tech A, 2001, 11(1):71-75.

10. Pinotti WH, Domene CE, Nasy A, et al. - "Video-assisted laparoscopy for the treatment of achalasia". In Peters JH, De Meester TR (eds) - Minimally invasive surgery of the foregut. St. Louis. Quality Medical Publishing, 1994, pp. 103-109.

11. Dor J, Humbert P, Dor V, et al. - L'intérêt de la technique de Nissen modifiée dans la prévention du reflux après cardiomyotomie extra-muqueuse de Heller. Mem Acad Chir, 1962, 88:881-883.

12. Collard JM, Romagnoli R, Lengele B, et al. - Heller - Dor procedure for achalasia: from conventional to video-endoscopic surgery. Acta Chir Belg, 1996, 96(2):62-65.

13. Mitchell PC, Watson DI, Devitt PG, et al. - Laparoscopic cardiomyotomy with a Dor patch for achalasia. Can J Surg, 1995, 38(5):445-448.

14. Peracchia A, Rosati R, Bona S, et al. - Laparoscopic treatment of functional diseases of the esophagus. Int Surg, 1995, 80(4):336-340.

15. Raiser F, Perdikis G, Hinder RA, et al. - Heller myotomy via minimal-access surgery: an evaluation of antireflux procedures. Arch Surg, 1996,131(6):593-598.

16. Delgado F, Bolufer JM, Martínez-Abad M, et al. - Laparoscopic treatment of esophageal achalasia. Surg Laparosc Endosc, 1996, 6(2):83-90.

17. Hunter JG, Trus TL, Branum GD, et al. - Laparoscopic Heller myotomy and fundoplication for achalasia. Ann Surg, 1997,225(6):655-665.

18. Patti MG, Pellegrini CA, Horgan S, et al. - Minimally invasive surgery for achalasia: an 8-year experience with 168 patients. Ann Surg, 1999, 230(4):587-595.

19. Zaninotto G, Costantini M, Molena D, et al. - Treatment of esophageal achalasia with laparoscopic Heller myotomy and Dor partial anterior fundoplication: prospective evaluation of 100 consecutive patients. J Gastrointest Surg, 2000, 4(3):282-289.
20. Lind JF, Burns CM, MacDougall JT - "Physiologic” repair for hiatus hérnia - manometric study. Arch Surg, 1965, 91:233237

21. Pinotti HW, Gama-Rodrigues JJ, Ellenbogen G, et al. - Nova técnica no tratamento cirúrgico do megaesôfago. Esofagocardiomiotomia associada à esofagofundogastropexia. Rev Goiana Méd, 1974, 20(1):1-8.

22. Pinotti HW, Felix VN, Domene CE, et al. - Recurrence of dysphagia in patients operated on for megaesophagus: analysis of determining factors. Chir Gastroenterol, 1979,13(1):1-7.

23. Pinotti HW, Felix VN, Raia A - O problema da recidiva da disfagia após tratamento do megaesôfago. Rev Goiana Méd, 1979, 25:191-198.

24. Rosati R, Fumagalli U, Bona S, et al. - Evaluating results of laparoscopic surgery for esophageal achalasia. Surg Endosc, 1998,12(3):270-2733.

25. Azevedo JLMC, Boulez J - Comparison between laparoscopic esophagocardiomyotomy with and without antireflux procedure in the treatment of achalasia. Surg Endosc, 2001, 15(Suppl 1):S54.

26. Boulez J, Meeus P, Espalieu P - Oesocardiomyotomie de Heller sans anti-reflux par voie laparoscopique. Analyse d'une serie de 27 cas. Ann Chir, 1997, 51(3):232-236.

27. Richards WO, Sharp KW, Holzman MD - An antireflux procedure should not routinely be added to a Heller myotomy. J Gastrointest Surg, 2001, 5(1):13-16.

28. Wang PC, Sharp KW, Holzman MD, et al. - The outcome of laparoscopic Heller myotomy without antireflux procedure in patients with achalasia. Am Surg, 1998, 64(6):515-521.

29. Igci A, Muslumanoglu M, Dolay K, et al. - Laparoscopic esophagomyotomy without an antireflux procedure for the treatment of achalasia. J Laparoendosc Adv Surg Tech A, 1998, 8(6):409-416.

30. Robertson GS, Lloyd DM, Wicks AC, et al. - Laparoscopic Heller's cardiomyotomy without an antireflux procedure. Br J Surg, 1995, 82(7):957-959.

31. Kjellin AP, Granqvist S, Ramel S, et al. - Laparoscopic myotomy without fundoplication in patients with achalasia. Eur J Surg, 1999, 165(12):1162-1166.

32. Azevedo O, Azevedo JLMC, Kozu FO, et al. - Wound healing of laparoscopic esophageal myotomy with and without an added Dor's fundoplication. International Congress of the European Association for Endoscopic Surgery, 12, 9 - 12 June 2004, Barcelona, Spain. Final Programme, O156, pp. 87.

33. McAdams AJ, Meikle AG, Taylor JO - One layer or two layer colonic anastomoses ? Am J Surg, 1970,120(4):546-550

34. Lyass S, Thoman D, Steiner JP, et al. - Current status of an antireflux procedure in laparoscopic Heller myotomy. Surg Endosc, 2003,17(4):554-558.

35. Ellis FH, Gibb SP - Reoperation after esophagomyotomy for achalasia of the esophagus. Am J Surg, 1975, 129(4):407-412.

Endereço para correspondência:

Prof Dr João Luiz M. C. Azevedo

Universidade Federal de São Paulo

Setor de Videocirurgia - Prédio da Cirurgia Experimental.

Rua Botucatu, 740 - V. Clementino

CEP 04023-900, São Paulo - SP.

Telfax: (11) 5576-4272

E-mail: jozevedo.dcir@epm.br

Site: http://www.cirurgiaonline.med.br 